\title{
Impact of COVID-19 pandemic on use of rituximab among children with difficult nephrotic syndrome
}

(c) The Author(s), under exclusive licence to the International Pediatric Research Foundation, Inc 2021

Pediatric Research (2022) 92:3-5; https://doi.org/10.1038/s41390021-01744-8

\section{INTRODUCTION}

Identifying the appropriate patient groups who will benefit most from shielding (strict social distancing/isolation) during the COVID19 pandemic is important for children as the mental health sequel of shielding are not inconsequential. ${ }^{1,2}$ Studies have indicated that elderly and adults with associated co-morbidities (hypertension, diabetes, chronic kidney disease and immunosuppressant drugs) are at higher risk in comparison to children. ${ }^{3}$ In contrast to adults where concomitant immunosuppression has been found to worsen the outcome of SARS-CoV-2 infection ${ }^{3,4}$ paediatric systematic reviews have concluded that SARS-CoV-2 infections occur in low numbers among immunocompromised children and overall have a favourable outcome. ${ }^{3,5}$ Literature is limited on the impact of SARS-CoV-2 infection among children with kidney disorders who are on potent immunosuppressant. ${ }^{6-8}$ Rituximab (B-lymphocyte depleting monoclonal antibody) is one such potent immunosuppressant frequently used in childhood difficult to treat nephrotic syndrome (cDNS) including steroid-dependent nephrotic syndrome (SDNS) and steroid-resistant nephrotic syndrome (SRNS). ${ }^{9}$ Medical literature on its use during the COVID-19 pandemic is scarce and safety remains a concern. ${ }^{7,8}$ With this perspective we undertook a questionnaire-based study to evaluate the effect of COVID-19 pandemic on the use of rituximab for cDNS ( $\leq 18$ years) and assess short-term outcomes among those testing positive for SARS-CoV-2 after receiving rituximab during the pandemic.

\section{METHODS}

A questionnaire was distributed to members of national and international paediatric nephrology societies (European Society of Pediatric Nephrology, Asian Society of Pediatric Nephrology and International Pediatric Nephrology Association) as well as circulated on Pedneph listserv. Since anonymized data were submitted formal ethical approval was not required. Data governance procedures were followed in the participating institutions. Continuous variables were expressed as median with Interquartile Range (IQR). Kruskal-Wallis test was used to compare between groups and chi-square was used to compare proportions.

\section{RESULTS}

Eighty-two responses from 33 countries that covered the pandemic period (February 2020 onward) were received. Only 9 respondents reported not using rituximab in their paediatric nephrology practice pre-pandemic and the primary reasons were either non-affordability due to high cost or non-availability of rituximab in their region. During the pandemic, $78 \%(57 / 73)$ of the respondents who were using rituximab pre-pandemic continued to use it. The remaining patients did not use rituximab for various reasons: concerns due to increased risk of immunosuppression $(n=9)$, lacking the need to use rituximab $(n=6)$ and disruption in the availability of rituximab $(n=1)$.

Four hundred and thirty-six children received 701 doses of Rituximab (SDNS $=80 \% ; n=347, \quad$ SRNS $=14 \% ; n=59 ; \quad$ and unknown $=7 \% ; n=30$ ) during the COVID-19 pandemic. Most centres $(81 \%, 46 / 57)$ did not modify their protocol for rituximab dosing and administration. Among those who modified their protocol six respondents modified their threshold for giving rituximab i.e. no longer used it for frequent relapses, two respondents no longer repeated rituximab on CD19 count normalisation, and three respondents changed the interval between the two doses of rituximab during COVID-19 pandemic. Concomitant immunosuppression was continued by $75 \%(n=43)$ of respondents post rituximab during the pandemic continued to use co-immunosuppressant post rituximab. A number of respondents $(24 / 73=33 \%)$ reported initial hesitancy but recommenced using rituximab later during the pandemic. The median number of children receiving rituximab per centre dropped 1 per month (IQR: $0.5-2$ ) prior to the pandemic to 0.3 per month (IQR: $0.2-0.9$ ); $p=$ 0.01 during the pandemic.

Of 436 children reported to receive rituximab during the pandemic; 30 (7\%) subsequently tested positive for SARS-CoV-2 (67\% male and median age: 10.2; IQR: $9.1-16.8$ years) at a median interval of 4 (IQR: 2.5-6.8) months post rituximab. These children received a median of 2 (IQR: $1-3$ ) doses of rituximab and $87 \%$ (26/ 30) were receiving concurrent immunosuppressant at the time of testing positive for COVID-19 (Fig. 1). The clinical outcome of children who tested positive for SARS-CoV-2 after receiving rituximab is detailed in Table 1 . Among those who tested positive for SARS-COV2, 63\% (19/30) were symptomatic and 37\% (11/30) required hospitalisation. Among symptomatic children, two-thirds had cough and fever and one-third also had an additional symptom of respiratory distress. Of note respiratory support was required in 6 of the 11 hospitalised children including 4 who required BiPAP among which 2 subsequently required mechanical 


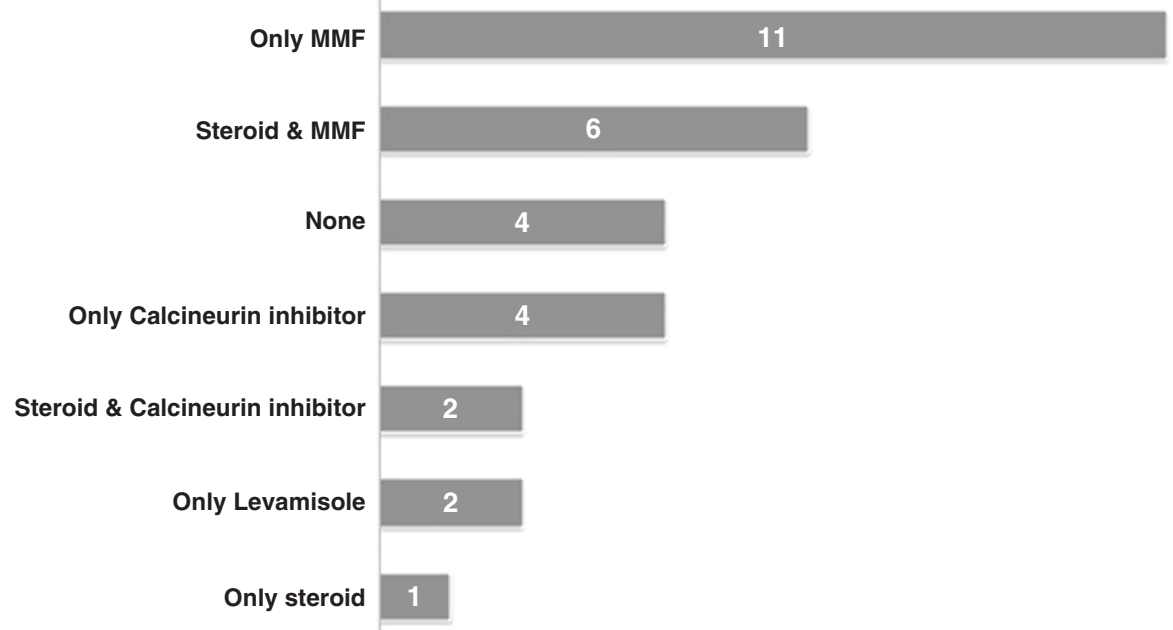

Fig. 1 Immunosuppressive co-medication at time of testing positive for SARS-CoV-2. MMF Mycophenolate mofetil.

Table 1. Clinical outcome of 30 children who tested positive for SARSCoV-2 following rituximab.

Clinical outcome of 30 children who tested positive for SARS-CoV-2 following rituximab $(n=30)$

Symptomatic [isolated cough: 1 , isolated fever: 4 , cough and fever: 8, cough, fever and respiratory distress: 6]

Hospitalisation

Required respiratory support [oxygen: 3, BiPAP: 1, BiPAP

$11(37 \%)$ and ventilator: 2]

BiPAP Bi-level positive airway pressure.

ventilation (Table 1). The commonly reported immunosuppressive management was to reduce or stop mycophenolate mofetil and switch to stress dose corticosteroids. Methylprednisolone was used in the two children requiring ventilation who were also given remdesevir. All children survived the infection; one child developed acute kidney injury but did not require dialysis.

\section{DISCUSSION}

The role of rituximab in paediatric nephrology is rapidly expanding and it is increasingly being advocated for cDNS. ${ }^{9}$ Given its marked impact on the immune system concerns regarding its use during any infectious pandemic such as the current COVID-19 pandemic cannot be ignored. The current survey, covering a large number of cDNS across many countries demonstrated a low incidence of these children subsequently testing positive for COVID-19 infection after receiving rituximab during the pandemic. This is similar to the observation reported in a nationwide prospective survey conducted by Shaunak et al. among immunocompromised children and young adults during the first wave of SARS-CoV-2 in the United Kingdom. ${ }^{10}$ Out of 1490 immunocompromised children across 46 centres, 110 children had symptoms consistent with SARS-CoV-2 infection but none tested positive. ${ }^{10}$ Reports on the impact of the use of rituximab in CDNS during the COVID-19 pandemic are scarce and the risk of developing SARS-CoV-2 infection post rituximab is unclear. In a previous international survey of COVID-19 infection among 113 children with kidney disease receiving various immunosuppressants, only $11(10 \%)$ received rituximab. ${ }^{7}$ Similarly in an Italian cohort of 1116 children with kidney disease and receiving immunosuppressants only $34(6 \%)$ children had received rituximab in the prior 6 months and overall only 3 children tested positive for COVID-19. Angeletti et al. focused on the use of rituximab and SARS-CoV-2 infection. ${ }^{8}$ Out of their cohort of 159 children who had received rituximab for difficult nephrotic syndrome none tested positive however the majority of them had received rituximab prior to the start of the pandemic.

The current survey reports clinical outcomes in one of the largest cohorts of CDNS receiving rituximab during the COVID-19 pandemic collected to date and highlights several important observations. Firstly, despite the COVID-19 pandemic, rituximab continued to play an important role in the management of cDNS worldwide as the majority of paediatric nephrologists (78\%) continued its use even during the pandemic. Secondly, in line with previous studies on the impact of the COVID-19 pandemic on immunocompromised children, ${ }^{3-6}$ the proportion of children on rituximab affected by SARS-CoV-2 continued to be low (6\%)and mirrored those reported from population-based data. Thirdly, the courses of the infection in this cohort were similar to the previously reported 102 children who received various immunosuppressants other than Rituximab ${ }^{7}$ with a comparable rate of hospitalisation ( $46 \%$ vs. $60 \%, p>0.05)$, although a trend towards the higher need for respiratory support among those hospitalised post rituximab (54\% vs 34\%; $p>0.05$ ) was observed. Most importantly, all children with SARS-COV2 infection under Rituximab treatment survived the disease.

We need to acknowledge the number of limitations in our study, of which the most important remains the inherent bias of conducting a study based on a retrospective questionnaire. It also cannot be ruled out that the wider asymptomatic testing capability affected our results as our survey was conducted one year after the start of the pandemic. Also, clinical practice at many contributing centres involved the administration of rituximab in combination with other immunosuppressants, rendering the assessment of the specific impact of rituximab on COVID-19 outcome difficult. Even comparing to immunocompromised children not exposed to rituximab by extracting data from the previous study ${ }^{7}$ did not solve this conundrum due to differences in underlying diseases and extent of immunosuppression. Lastly, as we received anonymous data, the possibility of duplicate reporting is a genuine concern although unlikely as in the 30 children who tested positive for COVID-19, additional clinical data were solicited, which helped us in ruling out data duplications.

In conclusion, despite the COVID-19 pandemic, rituximab continues to play an important role in the management of cDNS. In comparison to reports on the general paediatric population ${ }^{11}$ 
neither the risk of acquiring SARS-CoV-2 infection nor the risk of symptomatic disease and dismal outcomes appear to be increased among patients with cDNS receiving rituximab.

Rajiv Sinha ${ }^{1 凶}$, Matko Marlais $^{2}$, Subhankar Sarkar ${ }^{1}$, Varvara Obukhova ${ }^{3}$, Laura Lucchetti ${ }^{4}$, Anil Vasudevan ${ }^{5}$, Diana Carolina Chacon Jaimes ${ }^{6}$, Donald J. Weaver Jr $^{7}$, Małgorzata Stańczyk ${ }^{8}$, Mercedes Lopez-Gonzalez ${ }^{9}$, Franz Schaefer ${ }^{10}$ and Kjell Tullus ${ }^{2}$

${ }^{1}$ Institute of Child Health, Kolkata, India. ${ }^{2}$ Great Ormond Street Hospital, London, UK. ${ }^{3}$ Nephrology Department, Veltischev Research and Clinical Institute for Pediatrics of the Pirogov Russian National Research Medical University, Moscow, Russia. ${ }^{4}$ Bambino Gesù Children Hospital, Rome, Italy. ${ }^{5}$ St Johns Hospital, Bengaluru, India. ${ }^{6}$ Clinica FOSCAL, Bucaramanga, Colombia. ${ }^{7}$ Atrium Health Levine Children's Hospital, Charlotte, NC, USA. ${ }^{8}$ Polish Mother's Memorial Hospital Research Institute of Lodz, Łódź, Poland. ${ }^{9}$ University Hospital Valld'Hebron, Barcelona, Spain. ${ }^{10}$ Heidelberg University Hospital, Heidelberg, Germany. ${ }^{凶}$ email: rajivsinha_in@yahoo.com

\section{REFERENCES}

1. Green, P. Risks to children and young people during covid-19 pandemic. BMJ 369, m1669 (2020).

2. Tse, Y. et al. COVID-19: experiences of lockdown and support needs in children and young adults with kidney conditions. Pediatr. Nephrol. 36, 2797-2810 (2021).

3. Viner, R. M. et al. Susceptibility to SARS-CoV- 2 infection among children and adolescents compared with adults: a systematic review and meta-analysis. JAMA Pediatr. 175, 143-156 (2021).

4. Belsky, J. A. et al. COVID-19 in immunocompromised patients: a systematic review of cancer, hematopoietic cell and solid organ transplant patients. J. Infect. 82, 329-338 (2021)

5. Minotti, C., Tirelli, F., Barbieri, E., Giaquinto, C. \& Donà, D. How is immunosuppressive status affecting children and adults in SARS-CoV-2 infection? A systematic review. J. Infect. 81, e61-e66 (2020).

6. Mastrangelo, A. et al. Impact of COVID-19 pandemic in children with CKD or immunosuppression. Clin. J. Am. Soc. Nephrol. 16, 449-451 (2021).

7. Marlais, M. et al. COVID-19 in children treated with immunosuppressive medication for kidney diseases. Arch. Dis. Child. 106, 798-801 (2020).

8. Angeletti, A. et al. COVID-19 in children with nephrotic syndrome on anti-CD20 chronic immunosuppression. Clin. J. Am. Soc. Nephrol. 15, 1494-1495 (2020).

9. Trautmann, A. et al. IPNA clinical practice recommendations for the diagnosis and management of children with steroid-resistant nephrotic syndrome. Pediatr. Nephrol. 35, 1529-1561 (2020).

10. Shaunak, M. et al. ImmunoCOVID19 study group. COVID-19 symptom surveillance in immunocompromised children and young people in the UK: a prospective observational cohort study. BMJ Open 11, e044899 (2021).
11. Dong, Y. et al. Epidemiological characteristics of 2143 pediatric patients with 2019 coronavirus disease in China. Pediatrics https://doi.org/10.1542/peds.2020070 (2020).

\section{ACKNOWLEDGEMENTS}

We would like to thank Dr Abhijit Saha, Dr Alvero Madrid, Dr Amit Aggrawal, Dr Amrish Jain, Dr Ana Teixeira, Dr Angelus Marbella, Dr Anshuman Saha, Dr Antonia Bouts, Dr Catherine Quinlan, Dr Colette Girardin, Dr David Milford, Dr D.D. Torrace, Dr Dmitry Samsonov, Dr Faris Hashim, Dr Franklin Loachamin, Dr Gonzalo Myoga, Dr Hee Gyung Kyeng, Dr Ida Maria Schmidt, Dr Ifeoma Anochi, Dr Indira Agarwal, Dr Jean Grandy, Dr Jens König, Dr Jerome Harambat, Dr Jyoti Bagla, Dr Jyoti Sharma, Dr Karen Previdi Olandoski, Dr Keisuke Sugimoto, Dr L.P. Resontoc, Dr Luis Bofill, Dr Marta Melgosa, Dr M.K. Namgoong, Dr Manoj Matnani, Dr Manjusha Arumadi, Dr Motoshi Hatori, Dr Markus Kemper, Dr Manjusha Arumadi, Dr Mayatas, Dr Martin Bitzen, Dr Martin Kirschstein, Dr M.G. Penido, Dr Mimi Lhamu Mynak, Dr Naksa Hooman, Dr Nayoa Fujita, Dr O.P. Misra, Dr Oluseyi Oniyangi, Dr Paulina Alejandra, Dr Prashant Prabhu, Dr Prasad, Dr Quentin Bertrand, Dr Reynoso Angüis, Dr Salma Azarmeh, Dr Shankar Prasad Yadav, Dr Sumantra Raut, Dr Suprita Kalra, Dr Sushmita Banerjee, Dr Sukanya Govindam, Dr Shuji Kondo, Dr Selcuk Yuksel, Dr Scott Wenderfer, Dr Subal Pradhan, Dr Takayuki Okamoto, Dr Takeshi Ninchoji, Dr Tim Ulinski, Dr Valentina Gracchi, Dr Vela Belangero, Dr Velibor Tasic, Dr William Varade, Dr Yap Kim, Dr Yap Yok Chin, Dr Yiaw Kian Mun,Dr YincentTse, Dr Yuko Hamasaki and Dr Zubaida Rumana.

\section{AUTHOR CONTRIBUTIONS}

All the authors contributed to all of the following points: substantial contributions to conception and design, acquisition of data, or analysis and interpretation of data. Drafting the article or revising it critically for important intellectual content, and final approval of the version to be published.

\section{COMPETING INTERESTS}

The authors declare no competing interests.

\section{ADDITIONAL INFORMATION}

Correspondence and requests for materials should be addressed to Rajiv Sinha.

Reprints and permission information is available at http://www.nature.com/ reprints

Publisher's note Springer Nature remains neutral with regard to jurisdictional claims in published maps and institutional affiliations. 